\title{
Protein Markers for the Differential Diagnosis of Vascular Dementia and Alzheimer's Disease
}

\author{
A. H. Simonsen, ${ }^{1}$ N.-O. Hagnelius, ${ }^{2}$ G. Waldemar, ${ }^{1}$ T. K. Nilsson, ${ }^{3}$ and J. McGuire ${ }^{4}$ \\ ${ }^{1}$ Memory Disorders Research Group Section 6702, Department of Neurology, Copenhagen University Hospital Rigshospitalet, \\ Blegdamsvej 9, 2100 Copenhagen, Denmark \\ ${ }^{2}$ Department of Geriatric Medicine, Örebro University Hospital, 70185 Örebro, Sweden \\ ${ }^{3}$ Department of Laboratory Chemistry, Örebro University Hospital, 70185 Örebro, Sweden \\ ${ }^{4}$ Department of Incretin Biology, Novo Nordisk, Niels Steensens vej 1, 2820 Gentofte, Denmark
}

Correspondence should be addressed to A. H. Simonsen, anja.hviid.simonsen@rh.regionh.dk

Received 12 January 2012; Revised 11 March 2012; Accepted 3 April 2012

Academic Editor: John G. Marshall

Copyright ( 2012 A. H. Simonsen et al. This is an open access article distributed under the Creative Commons Attribution License, which permits unrestricted use, distribution, and reproduction in any medium, provided the original work is properly cited.

\begin{abstract}
Alzheimer's disease (AD) is the most common form of dementia found in all human populations worldwide, while vascular dementia $(\mathrm{VaD})$ is the second most common form of dementia. New biomarkers for early and specific diagnosis of $\mathrm{AD}$ and $\mathrm{VaD}$ are needed to achieve greater insight into changes occurring in the brain and direct therapeutic strategies. The objective of this explorative study was to discover candidate protein biomarkers for the differential diagnosis between VaD and AD. Surfaceenhanced laser desorption/ionization (SELDI) TOF-MS was used to differentially profile proteins and peptides in CSF samples from $28 \mathrm{AD}$ patients and 21 patients with $\mathrm{VaD}$. A combination of univariate (Kruskal-Wallis) and multivariate (independent component analysis) statistical approaches produced a list of 27 proteins and peptides that could differentiate between VaD and $\mathrm{AD}$. These markers represent various physiological processes, such as protein degradation (ubiquitin), protease inhibition (cystatin $\mathrm{C}$ and alpha-1-antichymoptrypsin), and inflammation (C3a and C4a) that are known to be represented in neurodegenerative diseases.
\end{abstract}

\section{Introduction}

Alzheimer's disease $(\mathrm{AD})$ is one of the most devastating brain disorders in the elderly. $\mathrm{AD}$ is a progressive neurodegenerative disease that represents the most common form of dementia today [1]. Age is the single most prominent risk factor with the incidence doubling every five years from the age of 65 [2].

Vascular dementia $(\mathrm{VaD})$ is a heterogeneous disorder that accounts for about $20 \%$ of all cases of dementia. Vascular dementia is characterized by neuronal death due to vascular lesions such as lacunar, cortical or subcortical infarcts, cerebral hemorrhage, and cardiogenic embolism, contributing to cognitive decline [3].

Vascular dementia and Alzheimer's disease frequently occur together and they may often act in combination to cause dementia and it is clinically challenging to separate these two diseases.
Biomarkers that can aid in the differential diagnosis between $\mathrm{VaD}$ and $\mathrm{AD}$ are relevant for several reasons. First, the pharmacological treatment strategy differs between the two diseases since patients with $\mathrm{VaD}$ only benefit modestly if at all from cholinesterase inhibitors and memantine which are the drugs used to treat AD. Since there are no approved drugs for the treatment of $\mathrm{VaD}$, current treatment is limited to the control of known vascular risk factors, such as hypertension and dyslipidemia [4]. Second, with new $\mathrm{AD}$ disease modifying drugs being tested it is crucial to differentiate between the patient groups for trial selection. Third, from an epidemiologic point of view it is of utmost importance that the clinical classification is based on the most up-to-date diagnostic practices in order to select the most appropriate predictor variables and monitoring tolls.

The aim of this explorative study was to discover candidate protein biomarkers for the differential diagnosis of $\mathrm{VaD}$ and $\mathrm{AD}$. 


\section{Materials and Methods}

2.1. Study Subjects and Samples. Patients for this study were recruited at University Hospital, Örebro, Sweden. They were all referred to the Memory Care Unit at the Department of Geriatrics for diagnostic assessment and treatment of suspected cognitive problems. According to Swedish research ethics law no proxy could sign the informed consent papers on behalf of the patient when collecting biobank material. All patients in the study group underwent a structured and thorough clinical investigation, including medical history, family history and socioeconomic data, and physical as well as neurological and psychiatric examination.

Considering the aim of this study, an important goal was to include cases with as narrowly defined diagnoses as possible in order to exclude cases with possible mixed $\mathrm{AD} / \mathrm{VaD}$, which would otherwise attenuate the differences between the two groups.

Blood chemistry tests were done on all (see below). Cerebrospinal fluid (CSF) samples were obtained by lumbar puncture at L3/L4 or L4/L5 level. The first 10-12 mL were collected in polypropylene tubes. The lumbar puncture and blood samples were collected at the same time, between 08 and $10 \mathrm{AM}$ after overnight fast. The samples were centrifuged and supernatants stored in a biobank freezer at $-80^{\circ} \mathrm{C}$.

The presence or absence of dementia was diagnosed according to Diagnostic and Statistical Manual of Mental Disorders (DSM-IV) criteria. Probable AD was diagnosed according to National Institute of Neurological and Communicative Diseases and Alzheimer's Disease and Related Disorders Association (NINCDS-ADRDA) criteria [5], VaD was diagnosed according to National Institute of Neurological Disorders and Stroke and Association Internationale pour la Recherché et l'Enseignement en Neurosciences (NINDSAIREN) criteria [3]. Disease severity was assessed using MMSE scores [6].

CSF samples (10-12 mL) were obtained by lumbar puncture, collected in polypropylene tubes, and gently mixed. The samples were centrifuged at $2000 \times \mathrm{g}$ for $10 \mathrm{~min}$ to remove cells and other insoluble material. Supernatants were frozen in aliquots and stored at $-80^{\circ} \mathrm{C}$. To avoid blood contamination, samples were discarded if they contained more than 500 erythrocytes per $\mu \mathrm{L}$.

The study was approved by the Regional Ethical Review Board, Uppsala. All patients gave informed consent to participate in the study, which was conducted in accordance with the provisions of the Helsinki Declaration.

2.2. Laboratory Methods. CSF samples were thawed and $5 \mu \mathrm{L}$ of each sample was diluted into $45 \mu \mathrm{L}$ of the appropriate binding buffer for each of the ProteinChip Array types (Ciphergen Biosystems, Fremont, CA, USA) using surfaceenhanced laser desorption/ionization time-of-flight mass spectrometry (SELDI-TOF-MS). The array types used were CM10 and IMAC30 coupled with nickel and H50. To ensure reproducibility of sample preparation and array analysis, a reference CSF standard was randomly distributed in several separate aliquots among the clinical samples and analyzed under exactly the same conditions. Reproducibility was measured by calculating average coefficients of variation (CV) for each set of acquisition parameters. All-array preparation was performed using a Biomek 2000 robot (Beckman Coulter) and randomized sample placement. The samples were allowed to bind for 60 minutes at room temperature. Each array was washed three times with the appropriate binding buffer and rinsed twice with water. Energy absorbing molecule (EAM) application was performed automatically using a modified Biodot AD3200 robot. Two aliquots of $0.75 \mu \mathrm{L}$ of solution containing $12.5 \mathrm{mg} / \mathrm{mL}$ sinapinic acid (SPA) in 50\% acetonitrile, $0.5 \%$ trifluoroacetic acid (TFA) were applied with drying in a controlled atmosphere between applications. The arrays were read at two different instrument settings to focus on lower and higher masses. Each sample was run in duplicates on separate arrays on successive robot runs. All arrays were analyzed using a SELDI-TOF MS ProteinChip Reader, series PCS4000 (Ciphergen Biosystems, Fremont, CA, USA). A protein profile was generated in which individual proteins were displayed within spectra as unique peaks based on their mass-to-charge ratio $(\mathrm{m} / \mathrm{z})$. Intensity normalization was done by calculating the total ion current for a spectrum, and the total ion current was then divided by the number of data points for that spectrum to obtain an average ion current for each individual spectrum. An external coefficient of 0.2 was chosen against which each spectrum was normalized. The very low mass region contains chemical "noise" from the matrix and was therefore excluded from the analysis.

\subsection{Purification and Identification of Candidate Markers.} Biomarkers were purified using combinations of chromatographic techniques employing a range of sorbents (Pall corporation, NY, USA) typically followed by SDSPAGE. One-quarter of a gel band was extracted using a solution containing 50\% formic acid, 25\% acetonitrile, $15 \%$ isopropanol, and $10 \%$ water and reanalyzed using the ProteinChip Reader to confirm their exact masses matched with the original biomarker. The remaining three-quarters of the band were in-gel digested with trypsin. Alternatively, whole bands of interest were extracted from gels and reanalyzed using the ProteinChip Reader to confirm their exact masses matched with the original biomarker. The gelextracted proteins were in-solution digested with trypsin. Tryptic digests were analyzed by peptide mapping using the ProteinChip Reader and by MS/MS using a Q-STAR XL tandem mass spectrometer (AB Sciex) fitted with a PCI-1000 ProteinChip Interface (Ciphergen Biosystems, Fremont, CA, USA). Biomarkers smaller than $4 \mathrm{kDa}$ were enriched by combinations of chromatographic techniques and identified directly by tandem MS without SDS-PAGE purification and/or trypsin digestion.

Peptide mass fingerprints and amino acid sequencing results were entered into the Mascot database (http://www .matrixscience.com) for protein identification.

2.4. Bioinformatics and Statistical Methods. ProteinChip profiling spectral data were collected using CiphergenExpress 
TABLE 1: Clinical Characteristics; values are means (SD).

\begin{tabular}{|c|c|c|c|c|c|c|c|c|c|}
\hline $\begin{array}{l}\text { Group (no. } \\
\text { samples) }\end{array}$ & $\begin{array}{c}\text { Gender } \\
\mathrm{M} / \mathrm{F}\end{array}$ & MMSE & Age years & $\begin{array}{c}\text { CSF } \\
\mathrm{A} \beta 1-42 \\
\mathrm{pg} / \mathrm{mL}\end{array}$ & $\begin{array}{c}\text { CSF total } \\
\text { Tau pg/mL }\end{array}$ & $\begin{array}{l}\text { CSF phos- } \\
\text { phorylated } \\
\text { Tau pg/mL }\end{array}$ & $\begin{array}{l}\mathrm{CSF} / \text { serum } \\
\text { albumin ratio }\end{array}$ & $\begin{array}{l}\mathrm{CSF} / \text { serum } \\
\text { folate ratio }\end{array}$ & $\begin{array}{c}\text { Total protein } \\
\text { g/L }\end{array}$ \\
\hline 28 probable $\mathrm{AD}$ & $10 / 18$ & $\begin{array}{l}20.0 \\
(3.7)\end{array}$ & $74.6(11.4)$ & 465 (146) & $682(316)$ & $91(36)$ & $5.9(1.7)$ & $2.5(0.9)$ & $0.38(0.11)$ \\
\hline $21 \mathrm{VaD}$ & $13 / 8$ & $\begin{array}{l}21.5 \\
(2.6)\end{array}$ & $76.2(8.4)$ & $634(169)$ & 389 (185) & $59(24)$ & $10.4(5.5)$ & $1.8(1.2)$ & $0.62(0.33)$ \\
\hline $\begin{array}{l}\text { Mann-Whitney } \\
P \text { value }\end{array}$ & & n.s. & n.s. & 0.011 & 0.0007 & 0.0021 & 0.0005 & 0.0348 & 0.002 \\
\hline
\end{tabular}

TABLE 2: Number of peaks and number of significant peaks on the three array surfaces and the two mass ranges used.

\begin{tabular}{lcccccc}
\hline Array type & \multicolumn{2}{c}{ CM10 } & IMAC30-Ni & & H50 \\
& Peaks & Significant peaks & Peaks & Significant peaks & Peaks & 38 \\
\hline $2-20 \mathrm{kDa}$ & 78 & 38 & 50 & 5 & 2 & 45 \\
$20-200 \mathrm{kDa}$ & 55 & 19 & 32 & 2 & 4 \\
\hline
\end{tabular}

data management software version 3.0 (Ciphergen Biosystems, Fremont, CA), where data handling and univariate analysis were also performed. All spectra were internally mass calibrated and peak intensities were normalized using total ion current.

All statistical analyses were conducted in R (http://www .r-project.org/). The data structure complexity and redundancy were reduced by identifying peak families (Spearman correlation coefficient $\geq 0.85$ ) and retaining only the most intense member. Comparisons were performed using a Mann-Whitney test with a Benjamini-Hochberg (BH) correction for multiple testing. A signal processing algorithm, independent components analysis (ICA), was applied to the data set in addition to the two group comparison [7].

ICA decomposes the data matrix (E) into a component matrix (C) comprised of the independent components (ICs) and a mixing matrix (A) giving the linear mixing of each IC in the samples. It is written as E = CA. An entry in the component matrix is denoted a load and for each IC the loads have a mean of zero and a standard deviation of one. The fastICA package [8] was used to decompose data by applying ICA and estimate the ICs. ICA was applied on data a total of five times and the results from each run were tested for stability by calculating an absolute correlation coefficient between the loads in each of the ICs with a set of ICs obtained from 100 independent runs of fastICA [9]. In each of the 100 runs, the maximum absolute correlation coefficient was saved and only components with a mean absolute correlation coefficient $\geq 0.80$ were retained. The capability of each IC to separate the samples according to classification was tested using a Mann-Whitney test with Benjamini-Hochberg correction for multiple testing. Peaks from significant components (adjusted $P$ value $<0.05$ ) having absolute loads greater than 5 times the standard deviation of the absolute loads were extracted as candidate peptide biomarkers.

\section{Results}

In this study we analyzed CSF samples from 28 patients with $\mathrm{AD}$ and 21 patients with $\mathrm{VaD}$ (see Table 1 for age, gender, and MMSE).

Neither age nor MMSE was significantly different between the $\mathrm{AD}$ and $\mathrm{VaD}$ groups with $P$ values of 0.71 and 0.28 for age and MMSE, respectively.

As expected, levels of Amyloid Beta 1-42 were significantly decreased in patients with $\mathrm{AD}$ and levels of Total Tau and Phosphorylated Tau were significantly increased in the same patient group compared to patients with VaD.

The albumin ratio was significantly higher in patients with $\mathrm{VaD}$ and the folate ratio was significantly lower for the same patient group as described before [10].

The SELDI intra-assay reproducibility of the discovery method was measured on median peak intensities in 48 individual spectra from a reference CSF sample and found to be between 14 and 19 percent CV (data not shown).

In Table 2 we describe the number of peaks found on the three SELDI array surfaces as well as the number of significant peaks. The CM10 surface had considerably more peaks, as well as significant peaks, which are summarized in Tables 3(a) and 3(b).

In total, eleven candidate biomarkers were obtained by a two-group comparison (Table 3(a)). Independent component analysis (ICA) gave rise to 21 significant components (Table 3(b)). There were six peaks in common between the two methods. ICA has the power to extract single variables or small groups of variables from a complex data set. The components can be analyzed for a particular characteristic, such as the ability to separate the two biological groups. The combination of this method with a classical univariate approach yielded a significant number of proteins to consider for further studies.

Unique peaks were found by analysis with ICA and Mann-Whitney, which emphasizes the complementary effect of combining feature reduction approaches. 
TABle 3: Candidate biomarkers and significant ICA components. Candidate biomarkers obtained from the univariate (a) and multivariate (b) analysis are listed separately. The $P$ values are Benjamini-Hochberg adjusted.

(a) Univariate analysis-Mann-Whitney

\begin{tabular}{|c|c|c|c|c|}
\hline $\mathrm{m} / \mathrm{z}$ & Array type & $P$ value & Direction of change in $\mathrm{AD}$ & ID \\
\hline 8295 & CM10 & 0,0017 & $\uparrow$ & Ubiquitin -3aa from CT \\
\hline 101801 & CM10 & 0,0075 & $\downarrow$ & \\
\hline 4352 & CM10 & 0,0142 & $\uparrow$ & Alpha-1-antichymotrypsin CT fragment \\
\hline 4745 & CM10 & 0,0142 & $\uparrow$ & \\
\hline 66676 & CM10 & 0,0142 & $\downarrow$ & Albumin \\
\hline 3515 & CM10 & 0,0181 & $\uparrow$ & Neuroendocrine protein 7B2 CT fragmen \\
\hline 17379 & CM10 & 0,0181 & $\downarrow$ & ApoA-II dimer \\
\hline 2840 & CM10 & 0,0186 & $\uparrow$ & \\
\hline 8614 & CM10 & 0,0274 & $\uparrow$ & C4a des-arg \\
\hline 4469 & CM10 & 0,0305 & $\uparrow$ & \\
\hline 2430 & CM10 & 0,0387 & $\uparrow$ & Integral Membrane 2B CT fragment \\
\hline
\end{tabular}

(b) Multivariate analysis-ICA

\begin{tabular}{|c|c|c|c|c|}
\hline $\mathrm{m} / \mathrm{z}$ & Array type & $P$ value & Direction of change in $\mathrm{AD}$ & ID \\
\hline 101801 & CM10 & 0,0067 & $\downarrow$ & \\
\hline 13105 & CM10 & 0,0067 & $\uparrow$ & \\
\hline 147523 & CM10 & 0,0067 & $\downarrow$ & $\operatorname{IgG}$ \\
\hline 16207 & CM10 & 0,0067 & $\downarrow$ & \\
\hline 17379 & CM10 & 0,0067 & $\downarrow$ & Apolipoprotein A-II dimer \\
\hline 28058 & CM10 & 0,0067 & $\downarrow$ & Apolipoprotein A-I \\
\hline 66676 & CM10 & 0,0067 & $\downarrow$ & Albumin \\
\hline 79578 & CM10 & 0,0067 & $\downarrow$ & Transferrin \\
\hline 8936 & CM10 & 0,0067 & $\uparrow$ & C3a des-arg \\
\hline 90294 & CM10 & 0,0067 & $\downarrow$ & \\
\hline 20934 & CM10 & 0,0072 & $\uparrow$ & Retinol binding protein \\
\hline 4745 & CM10 & 0,0072 & $\uparrow$ & \\
\hline 51402 & CM10 & 0,0077 & $\downarrow$ & \\
\hline 3515 & CM10 & 0,0104 & $\uparrow$ & Neuroendocrine protein 7B2 CT fragment \\
\hline 5262 & IM30Ni & 0,01076 & $\downarrow$ & \\
\hline 6973 & CM10 & 0,0159 & $\uparrow$ & \\
\hline 73422 & CM10 & 0,0182 & $\uparrow$ & \\
\hline 2840 & CM10 & 0,0255 & $\uparrow$ & \\
\hline 15124 & CM10 & 0,0347 & $\downarrow$ & \\
\hline 2249 & CM10 & 0,0467 & $\downarrow$ & \\
\hline 13358 & CM10 & 0,0471 & $\uparrow$ & Cystatin C \\
\hline
\end{tabular}

We found a total of 27 candidate markers; thirteen of the proteins have been identified and are therefore of special interest, see Tables 3(a) and 3(b). For representative spectra for the best marker found by Mann-Whitney and ICA, respectively, see Figures 1 and 2. Scatterplots of peak intensities of these two peaks can be seen in Figure 3 .

\section{Discussion}

In this exploratory study we aimed to discover potential biomarkers that could distinguish between $\mathrm{AD}$ and $\mathrm{VaD}$. Since it is important in an exploratory discovery design to avoid a type II error, we employed two different methods for feature selection [11-13] in order to obtain a more complete list of candidate markers.

In the literature other biomarkers indicating differences between $\mathrm{AD}$ and $\mathrm{VaD}$ have been described, such as albumin index [14] and the CSF/Serum folate ratio, where a reduced folate ratio was found to be a characteristic of dementia with vascular component [10].

In our sample set, there were statistically significant differences in CSF levels of amyloid $\beta 1-42$, total tau and phosphorylated tau between samples from patients with $\mathrm{VaD}$ and AD. However, several recently published large studies 


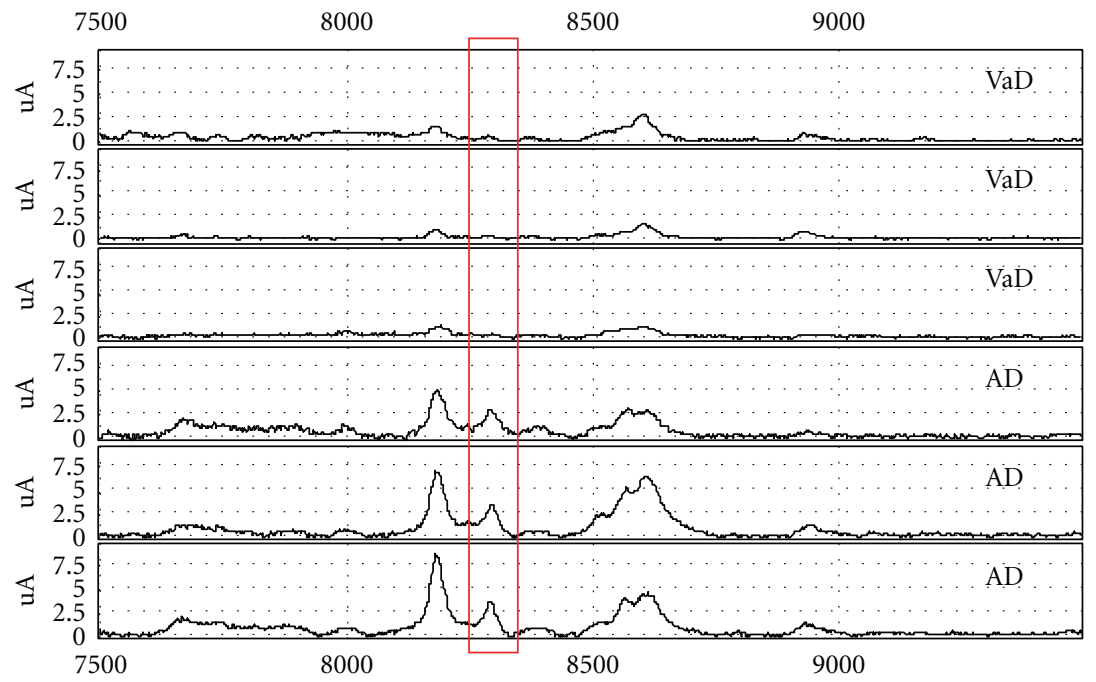

FIGURE 1: Representative spectra of Ubiquitin -3aa from CT on CM10 array.

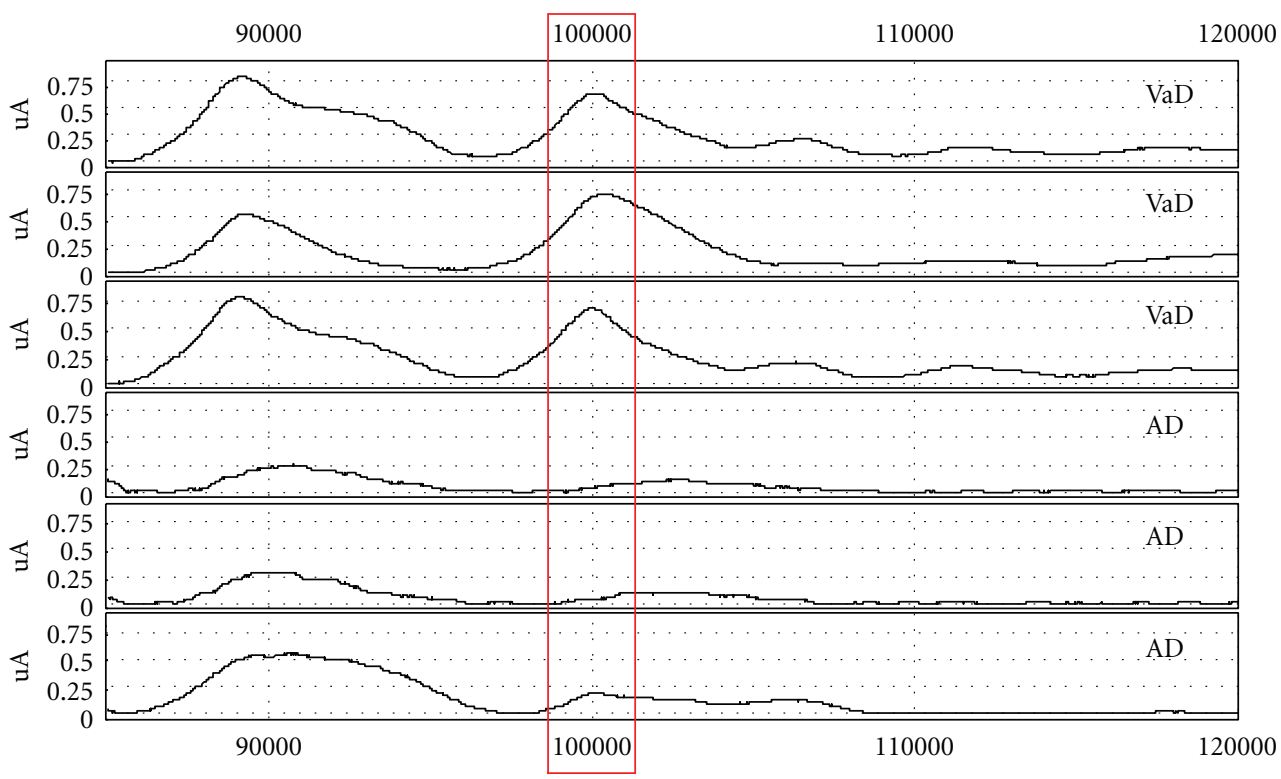

FIGURE 2: Representative spectra of $101 \mathrm{kDa}$ marker on CM10 array.
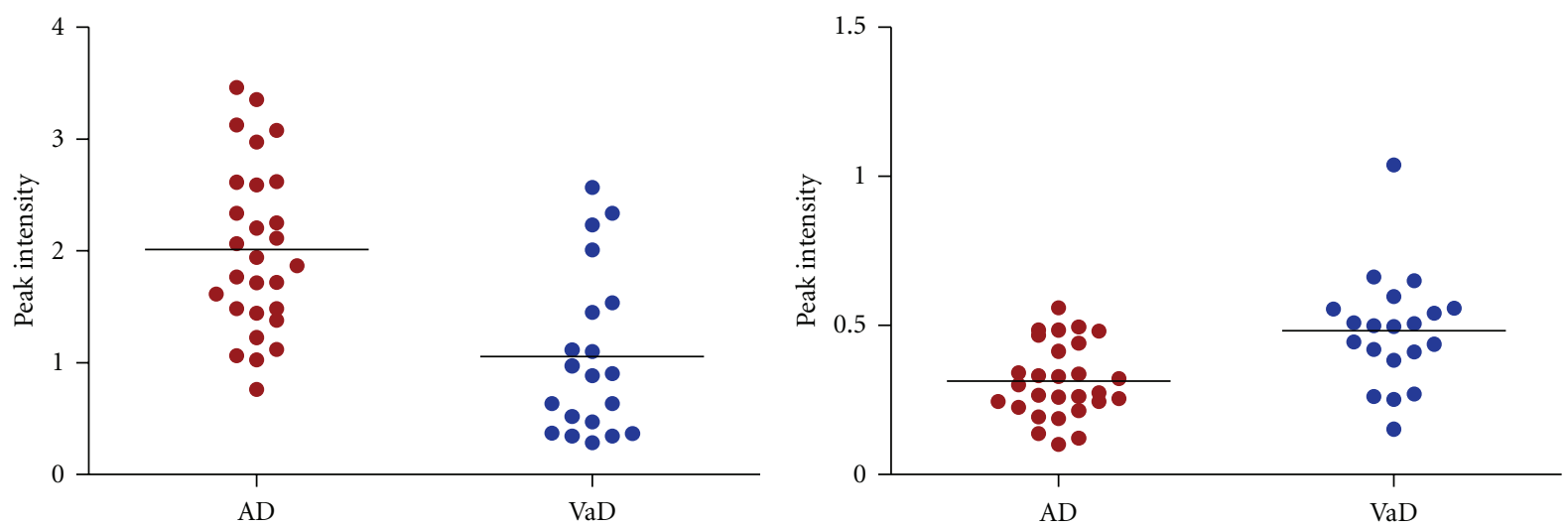

FIGURE 3: Scatter plot of peak intensities of Ubiquitin lacking 3 amino acids at the C-terminus left panel and the $101 \mathrm{kDa}$ marker right panel. 
report a substantial overlap between the levels in the VaD and AD groups [15].

We found a total of 27 candidate markers, thirteen of which have been identified. We found both up- and downregulated proteins in patients with $\mathrm{VaD}$ compared to $\mathrm{AD}$ even if the CSF protein concentration was significantly higher in the $\mathrm{VaD}$ group. This could be explained by the fact that we did find elevated levels of albumin, IgG, and transferrin, and these proteins comprise approximately $80 \%$ of the CSF proteome [16].

Several of the markers found in this study were recognized as markers that we have previously described in studies of $\mathrm{AD}$ biomarkers and markers for progression to $\mathrm{AD}$ from mild cognitive impairment (MCI) [17-19].

In our previous study we found decreased levels of the CT fragment of Alpha-1-antichymotrypsin in AD compared to healthy subjects [17]. Here we also found decreased levels of the same fragment in the CSF of patients with VaD compared to patients with AD. Thus it seems that levels of this protease inhibitor are lowest in patients with $\mathrm{VaD}$, followed by patients with $\mathrm{AD}$ and highest in healthy controls.

We have previously found increased levels of the Cterminal fragment of integral membrane $2 \mathrm{~B}$ in the CSF of $\mathrm{AD}$ and FTD patients compared to healthy controls [18], increased levels of the full length protein have also been described previously [20].

Integral membrane $2 \mathrm{~B}$ is also known as a BRI peptide, which is known to form insoluble deposits in the brains of patients with familial British and familial Danish dementia [21]. In this study we report reduced levels of the C-terminal fragment of integral membrane $2 \mathrm{~B}$ in the CSF of patients with $\mathrm{VaD}$ compared to $\mathrm{AD}$. Hence, the levels of this protein are similar in patients with $\mathrm{VaD}$ and healthy controls but can differentiate between patients with $\mathrm{VaD}$ and $\mathrm{AD}$.

In our earlier studies we found increased levels of the C3a peptide lacking the C-terminal arginine in the CSF of patients with $\mathrm{AD}$ compared to healthy controls and in patients with MCI who progressed to AD [17-19]. C3a is part of the complement system and involved in inflammatory processes. Here we show lower levels in the CSF of patients with $\mathrm{VaD}$ compared to patients with $\mathrm{AD}$. This means that the levels of $\mathrm{C} 3 \mathrm{a}$ des-arg in $\mathrm{VaD}$ are probably similar to the levels in healthy controls. Hence this marker can differentiate between $\mathrm{VaD}$ and $\mathrm{AD}$ but not between $\mathrm{VaD}$ and healthy aging.

We have previously reported decreased levels of the protease inhibitor Cystatin $\mathrm{C}$ in patients with $\mathrm{AD}$ compared to healthy controls $[17,18]$. In this study we find that the levels of Cystatin $\mathrm{C}$ are higher in patients with $\mathrm{VaD}$ than in patients with $\mathrm{AD}$. We postulate that the levels of Cystatin $\mathrm{C}$ are lowest in patients with $\mathrm{VaD}$, followed by patients with $\mathrm{AD}$, and highest in controls.

We have previously detected increased ubiquitin CSF levels in patients with MCI who progressed to AD [19]. Increased CSF ubiquitin levels have previously been found in both $\mathrm{AD}$ and $\mathrm{VaD}$ patients [22]. Here we show that $\mathrm{VaD}$ patients had significantly lower CSF concentrations of an ubiquitin fragment lacking three C-terminal amino acids compared to $\mathrm{AD}$ patients.
Neuroendocrine protein 7B2, also known as Secretogranin $\mathrm{V}$ is a molecular chaperone. To our knowledge it has not been previously described in CSF samples from patients with $\mathrm{AD}$ or $\mathrm{VaD}$. Mattson et al. described increased levels of Neuroendocrine protein 7B2 in the CSF of patients with frontotemporal dementia (FTD) with high expression of neurofilament [23] In this study we found decreased levels of this peptide in the CSF of $\mathrm{VaD}$ patients compared to $\mathrm{AD}$ patients.

ApoA-I levels in CSF have previously been found to be decreased in the CSF of $\mathrm{AD}$ patients when compared to healthy controls $[24,25]$; in this study we also find decreased levels in $\mathrm{AD}$ when comparing CSF from patients with $\mathrm{AD}$ to patients with $\mathrm{VaD}$.

In addition, we found increased levels of the dimer of ApoA-II in the CSF of patients with VaD compared to patients with $\mathrm{AD}$. In the literature increased levels of monomeric ApoA-II have been found in the CSF of patients with $\mathrm{AD}$ compared to healthy controls $[20,26]$. However, we were not able to detect the monomeric form of ApoA-II in our assay.

Higher levels of the complement pathway protein $\mathrm{C} 4 \mathrm{a}$ des-arg have been described previously in the CSF of patients with $\mathrm{AD}[20,26]$, and our group also found increased levels of the C4a peptide lacking the C-terminal arginine in MCI patients who progressed to AD [19]. Here we find higher levels of the C4a des-arg peptide in the CSF of patients with $\mathrm{AD}$ compared to $\mathrm{VaD}$.

Several studies have described albumin as being significantly up- or downregulated in the CSF of AD patients compared to healthy controls, see [27] for review. Here we found albumin to be lower in patients with $\mathrm{AD}$ compared to patients with $\mathrm{VaD}$ confirming the clinically obtained data on albumin ratios (Table 1). We also found lower levels of IgG in the CSF of patients with $\mathrm{AD}$ compared to $\mathrm{VaD}$. These two proteins are used clinically to evaluate blood-brain barrier impairment $[28,29]$, which is usually more impaired in $\mathrm{VaD}$ than $\mathrm{AD}$ and consistent with higher Albumin and IgG levels in the CSF of $\mathrm{VaD}$ patients.

Furthermore, we found lower levels of transferrin in $\mathrm{AD}$ that could perhaps similarly be explained by blood-brain barrier impairment in patients with $\mathrm{VaD}$.

Conflicting results of the CSF levels of retinol binding protein in patients with $\mathrm{AD}$ have been described. Increased levels were reported in $[20,24]$ and in MCI patients who progressed to $\mathrm{AD}$ [19] but decreased levels were found in [25]. In the present study we found lower levels of Retinol binding protein in the CSF of patients with $\mathrm{VaD}$ compared to $\mathrm{AD}$.

Presently, the occurrence of ischemic changes on computed axial tomography (CAT) scan is a very helpful tool to differentiate between $\mathrm{AD}$ and $\mathrm{VaD}$ patients. However, we believe that the candidate biomarkers found in this study could aid further in this differentiation.

We report both increased and decreased levels of several proteins and peptides when comparing the CSF from patients with $\mathrm{VaD}$ with the CSF from patients with $\mathrm{AD}$. These markers represent various physiological processes such as protein degradation (ubiquitin), protease inhibition 
(Cystatin C and Alpha-1-antichymotrypsin), and inflammation $(\mathrm{C} 3 \mathrm{a}$ and $\mathrm{C} 4 \mathrm{a})$ that are known to be represented in neurodegenerative diseases.

In summary, we found a total of 27 candidate markers for the diagnostic differentiation between $\mathrm{VaD}$ and $\mathrm{AD}$. Even for the markers with the lowest $P$ values there was considerable overlap between the two groups. Therefore we do not expect any of these markers to be used on its own but in combination with other markers such as the folate ratio, levels of Amyloid Beta 1-42, total Tau, and phosphorylated Tau. All of the thirteen markers identified in this study have been described previously in the literature in the CSF of patients with $\mathrm{AD}$ and MCI. To our knowledge, this is the first study where they are described in patients with $\mathrm{VaD}$.

A limitation of the present study is the lack of a healthy control group. Future studies should enroll pathology confirmed $\mathrm{AD}$ and $\mathrm{VaD}$ dementia cases as well as longitudinally followed control individuals. Furthermore the diagnostic performance of selected candidate markers from this study should be investigated in large prospective multicenter studies.

Despite the obvious application of these novel biomarkers to improve the accuracy of early diagnosis they could also play a role in the development of new disease modifying drugs. This and future proteomic and genomic explorations of clinical samples will likely play an important role in unraveling the biochemistry of dementia pathogenesis ultimately leading to new therapeutic targets.

\section{Acknowledgments}

This work was partially supported by funding from the Danish Diabetes Association (to J. McMGuire), Nyckelfonden, Örebro University Hospital (to N.-O. Hagnelius), The Lundbeck Foundation (Grant no. R32-A2833) (to G. Waldemar), and Simon Spies Fonden (to G. Waldemar). None of these funding sources had any role in the preparation of this paper or in the decision to submit it for publication.

\section{References}

[1] R. P. Friedland, "Alzheimer's Disease: clinical features and differential diagnosis," Neurology, vol. 43, pp. S45-S51, 1993.

[2] K. Ritchie, D. Kildea, and J. M. Robine, "The relationship between age and the prevalence of senile dementia: a metaanalysis of recent data," International Journal of Epidemiology, vol. 21, no. 4, pp. 763-769, 1992.

[3] G. C. Roman, T. K. Tatemichi, T. Erkinjuntti et al., "Vascular dementia: diagnostic criteria for research studies: report of the NINDS-AIREN International Workshop," Neurology, vol. 43, no. 2, pp. 250-260, 1993.

[4] A. Moretti, A. Gorini, and R. F. Villa, "Pharmacotherapy and prevention of vascular dementia," CNS and Neurological Disorders, vol. 10, no. 3, pp. 370-390, 2011.

[5] G. McKhann, D. Drachman, and M. Folstein, "Clinical diagnosis of Alzheimer's disease: report of the NINCDS-ADRDA work group under the auspices of Department of Health and Human Services Task Force on Alzheimer's disease," Neurology, vol. 34, no. 7, pp. 939-944, 1984.
[6] M. F. Folstein, S. E. Folstein, and P. R. McHugh, "'Mini mental state'. A practical method for grading the cognitive state of patients for the clinician," Journal of Psychiatric Research, vol. 12, no. 3, pp. 189-198, 1975.

[7] Y. Benjamini and Y. Hochberg, "Controlling the false discovery rate: a practical and powerful approach to multiple testing," Journal of the Royal Statistical Society B, vol. 57, no. 1, pp. 289300, 1995.

[8] A. Hyvarinen, "Fast and robust fixed-point algorithms for independent component analysis," IEEE Transactions on Neural Networks, vol. 10, no. 3, pp. 626-634, 1999.

[9] P. Chiappetta, M. C. Roubaud, and B. Torrésani, "Blind source separation and the analysis of microarray data," Journal of Computational Biology, vol. 11, no. 6, pp. 1090-1109, 2004.

[10] N. O. Hagnelius, L. O. Wahlund, and T. K. Nilsson, "CSF/serum folate gradient: physiology and determinants with special reference to dementia," Dementia and Geriatric Cognitive Disorders, vol. 25, no. 6, pp. 516-523, 2008.

[11] D. Mantini, F. Petrucci, P. Del Boccio et al., "Independent component analysis for the extraction of reliable protein signal profiles from MALDI-TOF mass spectra," Bioinformatics, vol. 24, no. 1, pp. 63-70, 2008.

[12] A. J. Overgaard, H. G. Hansen, M. Lajer et al., "Plasma proteome analysis of patients with type 1 diabetes with diabetic nephropathy," Proteome Science, vol. 8, article 4, 2010.

[13] H. G. Hansen, J. Overgaard, M. Lajer et al., "Finding diabetic nephropathy biomarkers in the plasma peptidome by highthroughput magnetic bead processing and MALDI-TOF-MS analysis," Proteomics, vol. 4, no. 8-9, pp. 697-705, 2010.

[14] A. Wallin, M. Sjögren, A. Edman, K. Blennow, and B. Regland, "Symptoms, vascular risk factors and blood-brain barrier function in relation to CT white-matter changes in dementia," European Neurology, vol. 44, no. 4, pp. 229-235, 2000.

[15] N. S. Schoonenboom, F. E. Reesink, N. A. Verwey et al., "Cerebrospinal fluid markers for differential dementia diagnosis in a large memory clinic cohort," Neurology, vol. 78, no. 1, pp. 47-54, 2012.

[16] E. J. Thompson and G. Keir, "Laboratory investigation of cerebrospinal fluid proteins," Annals of Clinical Biochemistry, vol. 27, no. 5, pp. 425-435, 1990.

[17] A. H. Simonsen, J. McGuire, V. N. Podust et al., "Identification of a novel panel of cerebrospinal fluid biomarkers for Alzheimer's disease," Neurobiology of Aging, vol. 29, no. 7, pp. 961-968, 2008.

[18] A. H. Simonsen, J. McGuire, V. N. Podust et al., "A novel panel of cerebrospinal fluid biomarkers for the differential diagnosis of Alzheimer's disease versus normal aging and frontotemporal dementia," Dementia and Geriatric Cognitive Disorders, vol. 24, no. 6, pp. 434-440, 2007.

[19] A. H. Simonsen, J. McGuire, O. Hansson et al., "Novel panel of cerebrospinal fluid biomarkers for the prediction of progression to Alzheimer dementia in patients with mild cognitive impairment," Archives of Neurology, vol. 64, no. 3, pp. 366-370, 2007.

[20] F. Abdi, J. F. Quinn, J. Jankovic et al., "Detection of biomarkers with a multiplex quantitative proteomic platform in cerebrospinal fluid of patients with neurodegenerative disorders," Journal of Alzheimer's Disease, vol. 9, no. 3, pp. 293-348, 2006.

[21] O. El-Agnaf, G. Gibson, M. Lee, A. Wright, and B. M. Austen, "Properties of neurotoxic peptides related to the BRI gene," Protein and Peptide Letters, vol. 11, no. 3, pp. 207-212, 2004.

[22] K. Blennow, P. Davidsson, A. Wallin, C. G. Gottfries, and L. Svennerholm, "First place. 1993 IPA Research Awards in Psychogeriatrics: ubiquitin in cerebrospinal fluid in Alzheimer's 
disease and vascular dementia," International Psychogeriatrics, vol. 6, no. 1, pp. 13-22, 1994.

[23] N. Mattsson, U. Rüetschi, Y. A. L. Pijnenburg et al., "Novel cerebrospinal fluid biomarkers of axonal degeneration in frontotemporal dementia," Molecular Medicine Reports, vol. 1, no. 5, pp. 757-761, 2008.

[24] P. Davidsson, A. Westman-Brinkmalm, C. L. Nilsson et al., "Proteome analysis of cerebrospinal fluid proteins in Alzheimer patients," NeuroReport, vol. 13, no. 5, pp. 611-615, 2002.

[25] M. Puchades, S. F. Hansson, C. L. Nilsson, N. Andreasen, K. Blennow, and P. Davidsson, "Proteomic studies of potential cerebrospinal fluid protein markers for Alzheimer's disease," Molecular Brain Research, vol. 118, no. 1-2, pp. 140-146, 2003.

[26] J. Zhang, D. R. Goodlett, and T. J. Montine, "Proteomic biomarker discovery in cerebrospinal fluid for neurodegenerative diseases," Journal of Alzheimer's Disease, vol. 8, no. 4, pp. 377-386, 2005.

[27] H. Zetterberg, U. Rüetschi, E. Portelius et al., "Clinical proteomics in neurodegenerative disorders," Acta Neurologica Scandinavica, vol. 118, no. 1, pp. 1-11, 2008.

[28] A. Wallin and M. Sjögren, "Cerebrospinal fluid cytoskeleton proteins in patients with subcortical white-matter dementia," Mechanisms of Ageing and Development, vol. 122, no. 16, pp. 1937-1949, 2001.

[29] I. Skoog, A. Wallin, P. Fredman et al., "A population study on blood-brain barrier function in 85-year-olds: relation to Alzheimer's disease and vascular dementia," Neurology, vol. 50, no. 4, pp. 966-971, 1998. 

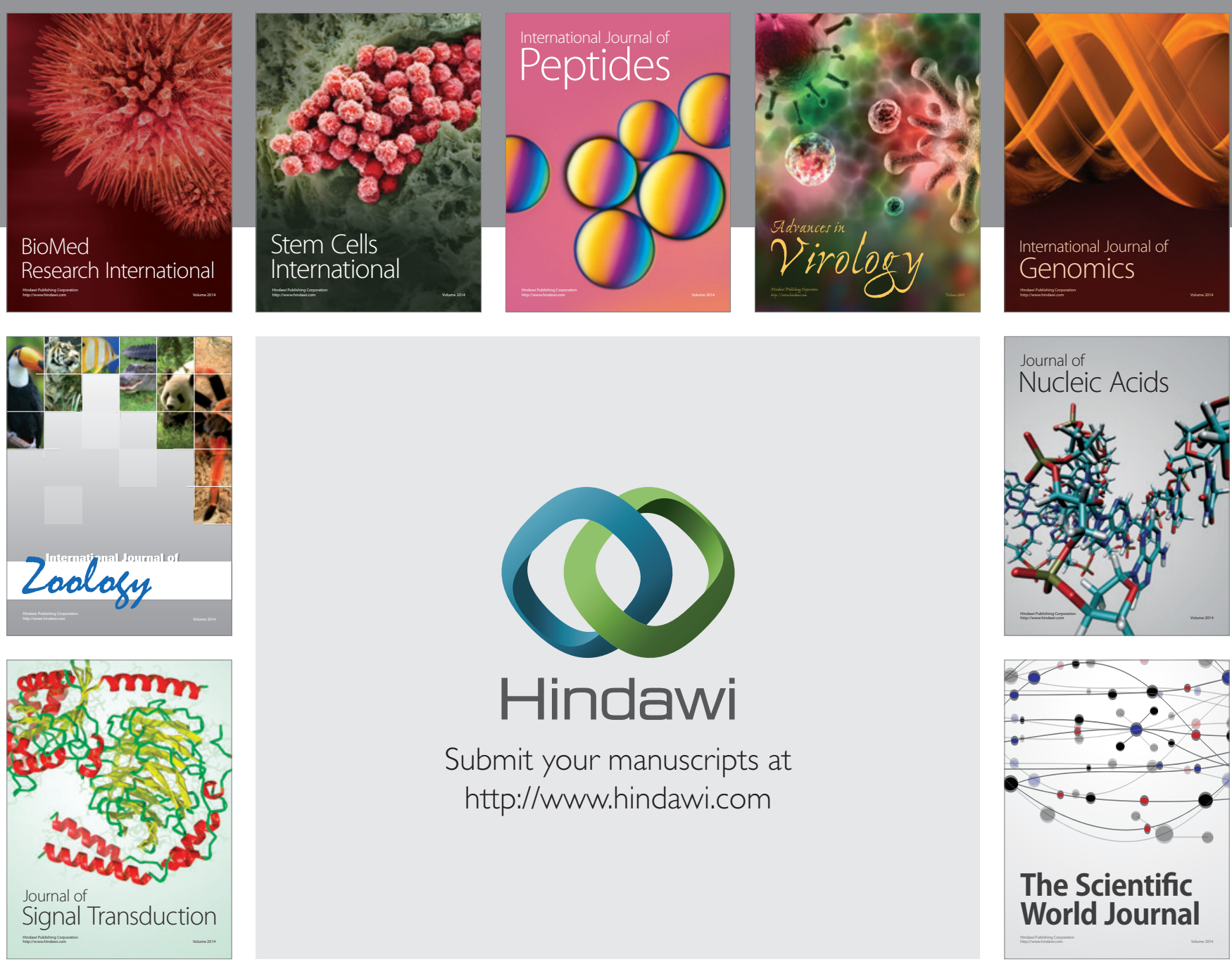

Submit your manuscripts at

http://www.hindawi.com
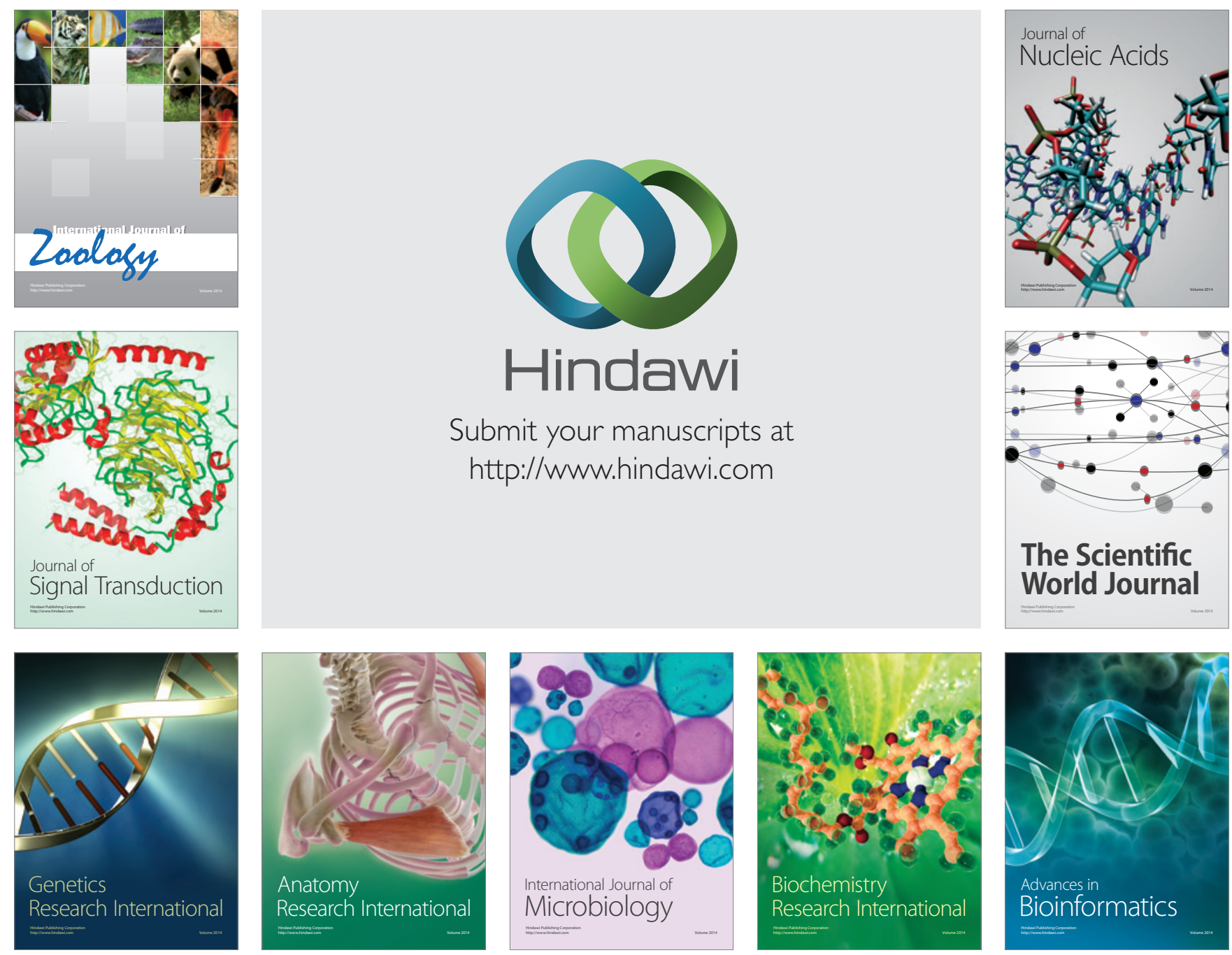

The Scientific World Journal
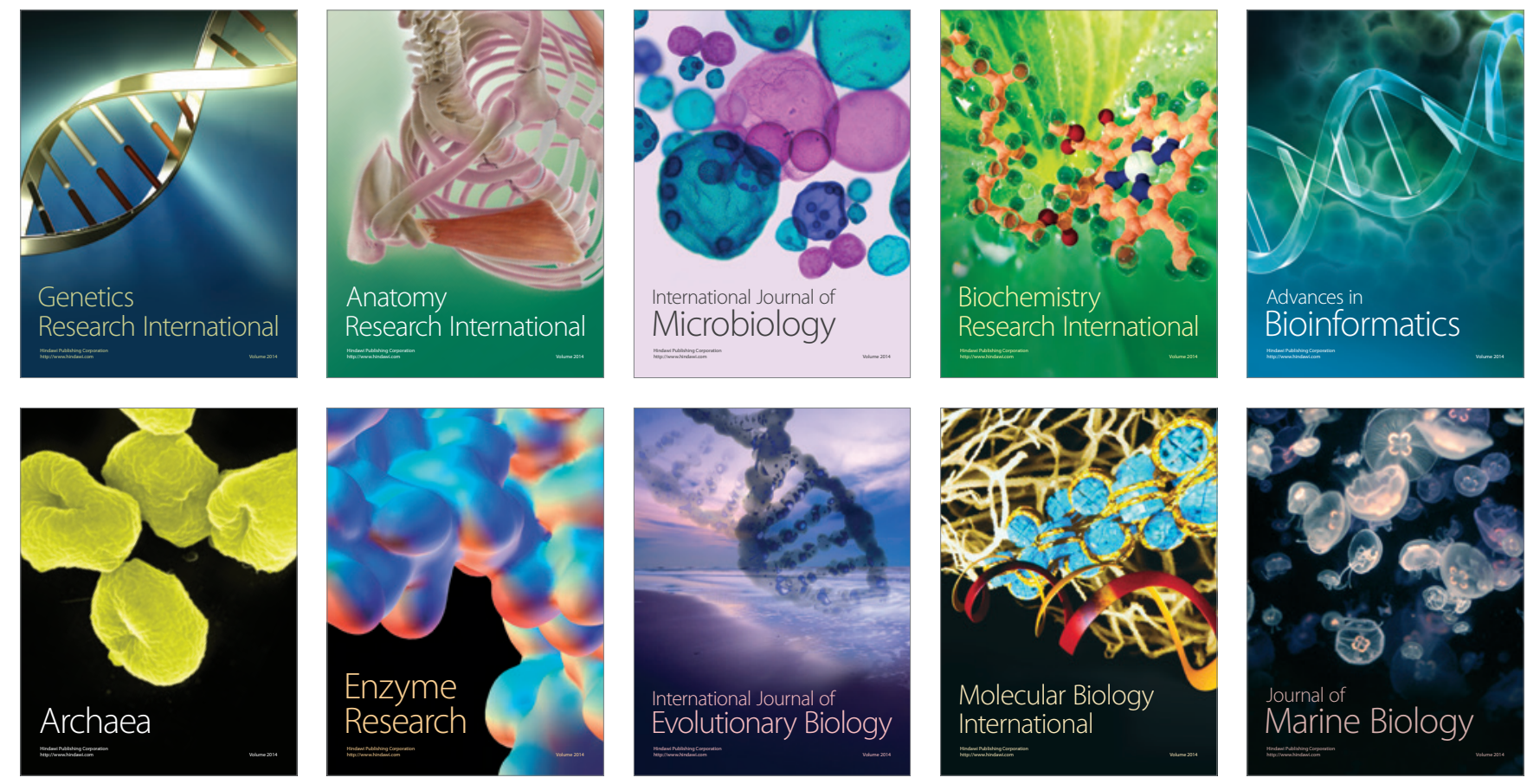